Onkologe $2018 \cdot 24: 797-801$ https://doi.org/10.1007/s00761-018-0400-5 Online publiziert: 28. Juni 2018

(c) Der/die Autor(en) 2018

CrossMark

\title{
Wolfgang Dörr
}

Klinik für Strahlentherapie, ATRAB - Applied and Translational Radiobiology, Medizinische Universität Wien, Wien, Österreich

\section{Spätfolgen nach Radiotherapie}

effects“, CLE) auftreten. So, wenn die früh reagierenden Gewebsanteile (z.B. Epithelien) eine Schutzfunktion gegen mechanische und/oder chemische Einflüsse haben, welche während der Frühreaktion beeinträchtigt wird, sodass zusätzliche Traumata auf die Zielstrukturen der Spätfolgen einwirken [7].

Parenchymzellen in jedem Organ werden durch Strahlenexposition inaktiviert. Daneben trägt bei Endothelzellen eine veränderte biologische Aktivität [10, 21, 23] zur Strahlenreaktion bei. Endothelzellvakuolisierung und Endothelablösung sind häufig schon zu sehr frühen Zeitpunkten zu beobachten, gefolgt vom Endothelzelltod. Zudem finden sich ein subendotheliales Ödem und die Bildung von Thromben [12]. Dies geht einher mit einer Leukozytenadhäsion und -infiltration in die Gefäßwand. Diese Veränderungen führen $\mathrm{zu}$ einem progressiven Verlust von Kapillaren, mit der Folge einer Parenchymatrophie.

Teleangiektasien werden in praktisch allen bestrahlten Geweben und Organen beobachtet. Diese beruhen ebenfalls auf der Schädigung der Endothelzellen, in Verbindung mit dem Verlust von glatten Muskelzellen bei größeren Kapillaren und Venen. Im Darm, in der Harnblase oder auch im ZNS können Teleangiektasien wegen der Blutungsneigung klinisch relevant sein; in der Haut sind sie ein kosmetisches Problem. Über längere Zeiträume können die teleangiektatischen Kapillaren kollabieren, was zu einer Reversibilität der Blutungen führt [13, 14].

In mitotischen Fibroblasten wird durch Strahlenexposition die Differenzierung zu postmitotischen Fibrozyten induziert, mit der Folge einer drastisch erhöhten Kollagensynthese [18]. Die re- sultierende Fibrose wirkt sich signifikant auf die Organfunktion aus $[1,15,28]$.

Makrophagen, bestrahlt oder nach Bestrahlung rekrutiert, tragen ebenfalls signifikant zu den relevanten Signalkaskaden bei. Reaktive Sauerstoff- und Stickstoffspezies, die chronisch von verschiedenen Zellpopulationen produziert werden, scheinen in Kombination mit chronischer Hypoxie und einer permanent aktivierten und modifizierten Kaskade von Zytokinen eine wesentliche Rolle bei der Pathogenese chronischer Strahlenfolgen zu spielen $[1,9,19,20]$.

Die geschilderten Veränderungen führen zu einer zunächst subklinischen, jedoch progressiven Parenchymschädigung und schließlich zum Funktionsverlust innerhalb desjenigen Organvolumens, das einer bestimmten Dosis ausgesetzt war. Die klinischen Konsequenzen hängen von der Architektur des Organs, der Strahlentoleranz und teilweise von der Lokalisation des exponierten Volumens im Organ ab [11].

\section{》) Das Organ definiert die klinische Manifestation bei Teilorganexposition}

Jede der beteiligten zellulären/geweblichen Komponenten reagiert auf eine Strahlenexposition mit einer spezifischen Dosisabhängigkeit; das Zusammenspiel definiert dann die Gesamtdosisantwort für die verschiedenen klinischen Endpunkte. Für verschiedene Organe kann die Relevanz der einzelnen pathogenetischen Komponenten unterschiedlich sein $[9,10,16]$.
Frühe und späte Strahleneffekte sind primär voneinander unabhängig. In bestimmten Fällen können jedoch konsekutive Spätfolgen („consequential late 


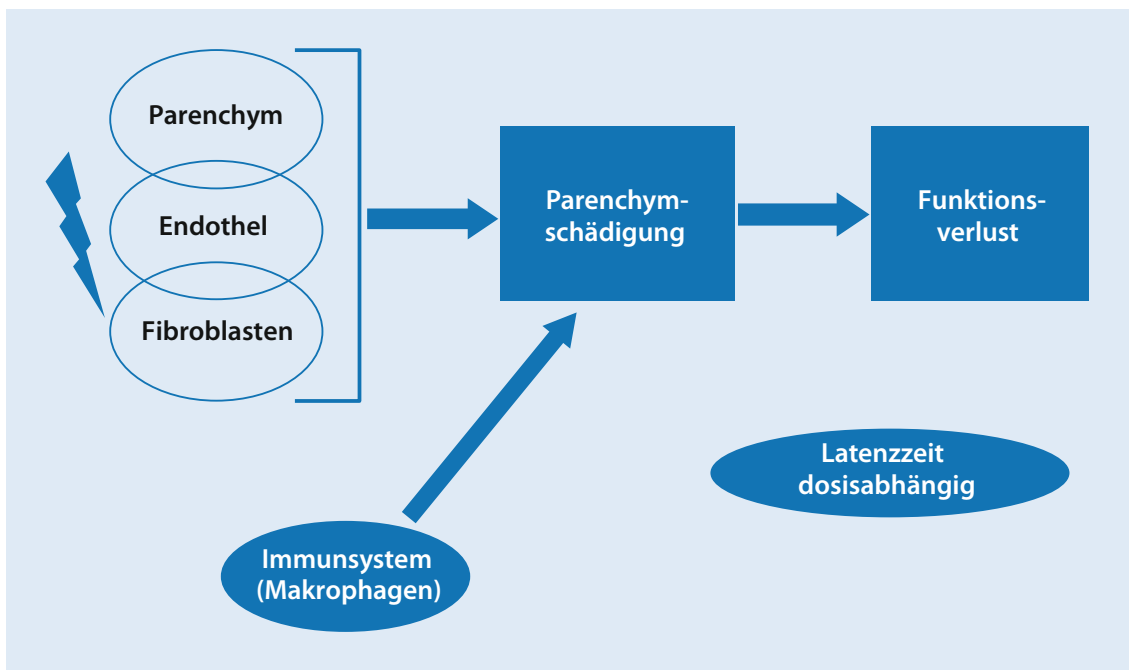

Abb. $1 \Delta$ Pathogenese von Spätfolgen - Komponenten und Abläufe

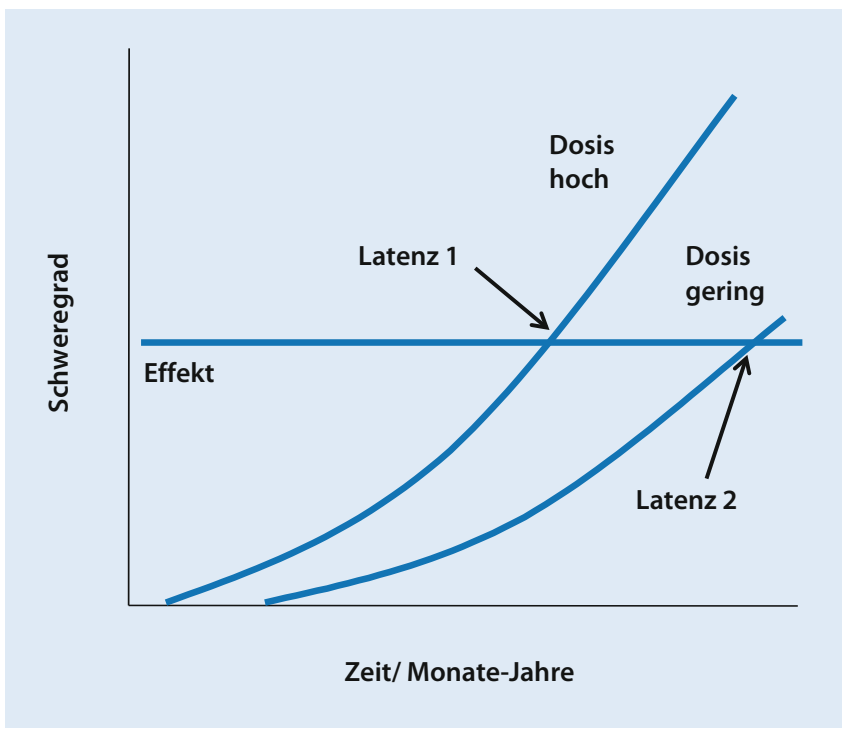

\section{Latenzzeiten}

Die Latenzzeiten für chronische Strahlenfolgen sowie die Progressionsrate sind invers dosisabhängig (• Abb. 2). Als Folge werden späte Normalgewebseffekte bei niedrigeren Strahlendosen erst mit zunehmenden Nachbeobachtungszeiten registriert.

\section{》) Latenzzeiten für Strahlen- spätfolgen sind invers dosisabhängig}

Somit ist - v. a. bei modernen Bestrahlungstechniken - eine ausreichend lange Nachsorge zur vollständigen Erfas-
Abb. $2<$ Verlauf von Spätfolgen Dosisabhängigkeit der Latenz

Haut und Hautanhangsgebilde

Die chronische subkutane Fibrose, die sich klinisch als Induration manifestiert, beruht überwiegend auf einem Anstieg der Kollagenfasern und einer Reduktion des Fettgewebes. Teleangiektasien beruhen auf den Gefäßeffekten in der Dermis.

$\mathrm{Ab}$ einer kumulativen Dosis von etwa 12 Gy wird ein Verlust in der Funktion der Talgdrüsen beobachtet, bei etwas höheren Dosen reagieren auch die Schweißdrüsen, was zu einer typischen „trockenen Haut" führt. In den Haarfollikeln führen Einzeldosen von etwa 4 bzw. 10 Gy $\mathrm{zu}$ vorübergehendem bzw. dauerhaftem Haarausfall. In fraktionierten Protokollen erlauben signifikant höhere Dosen bis zu 40 Gy noch ein Nachwachsen der Haare. Häufig finden sich Farbveränderungen der nachwachsenden Haare.

Die Okklusion von Lymphgefäßen, als direkte Strahlenwirkung sowie durch die Fibrose, kann zu einem chronischen Lymphödem führen, was sich ebenfalls signifikant auf die Lebensqualität der Patienten auswirkt.

\section{Lunge}

Die Lunge gehört zu den empfindlichen Organen. Neben einer Verringerung des bestrahlten Volumens sind reduzierte Dosen pro Fraktion am wirksamsten zur Verminderung/Vermeidung schwerer Lungenreaktionen. Klinische Symptome einer Strahlenpneumonitis sind eine verminderte Compliance, progressive Dyspnoe, verminderter Gasaustausch und trockener Husten, bis hin zum kardiorespiratorischen Versagen. gänglich [5]. Folglich erfordert aber auch die Definition von Toleranzdosen immer Informationen über die Nachbeobachtungszeit [5].

\section{Beispiele später Strahlenfolgen}

In diesem Abschnitt werden die Reaktion und die Toleranz einiger klinisch wichtiger Normalgewebe („organs at risk“, OAR) zusammengefasst. Ausführlichere Beschreibungen finden sich in QUANTEC [2], in den Berichten der Internationalen Strahlenschutzkommission ICRP [4] und in verschiedenen Übersichtsarbeiten (z. B. [5]).

\section{》) Lunge und Niere sind sehr strahlenempfindlich bei ausgeprägtem Volumeneffekt}

Fibrosen entwickeln sich langsam über einen Zeitraum von einigen Monaten bis zu Jahren. Lokale fibrotische Reaktionen müssen bei allen Patienten mit frühen Reaktionen erwartet werden, was auf eine starke konsekutive Komponente der Spätreaktion hinweist. Höheres Alter und eine Tamoxifenbehandlung erhöhen sig- 
nifikant die Häufigkeit der Pneumopathie [6].

\section{Niere}

Strahlenfolgen an der Niere entwickeln sich sehr langsam über viele Jahre. Die Strahlennephropathie manifestiert sich in der Regel als Proteinurie, Hypertonie und Beeinträchtigung der Urinkonzentrationsfunktion. Eine Anämie (Hämolyse, verminderte Erythropoetinproduktion) ist gewöhnlich vorhanden. Eine leichtgradige Nephropathie, in Form einer Proteinurie, kann über einen Zeitraum von vielen Jahren beobachtet werden. Nach partieller Nierenbestrahlung kann sich eine Hypertonie nach einer Latenzzeit von bis zu 10 Jahren entwickeln.

Eine glomeruläre Endothelschädigung gilt als initiales Ereignis, welches zur glomerulären Sklerose und später zur tubulointerstitiellen Fibrose führt. Durch den Verlust von tubulären Epithelzellen kann Fibrin in das Interstitium austreten, was den Beginn der Fibrose verursacht.

\section{Nervensystem}

Das Nervensystem ist relativ unempfindlich für eine Strahlenexposition, jedoch führt eine Schädigung zu schwerwiegenden klinischen Folgen. Die wichtigsten Strahlensyndrome im zentralen Nervensystem entwickeln sich wenige Monate bis mehrere Jahre nach der Therapie. Einige Reaktionen, die innerhalb der ersten 6 Monate auftreten können, umfassen eine vorübergehende Demyelinisierung („Somnolenzsyndrom“) oder eine Leukenzephalopathie. Die typische Strahlennekrose kann nach 6 Monaten, aber auch noch nach 2-3 Jahren auftreten. Histopathologisch sind Veränderungen, die innerhalb des ersten Jahres auftreten, meist auf die weiße Substanz beschränkt. Für Zeiträume jenseits von 6-12 Monaten zeigt gewöhnlich auch die graue Substanz Veränderungen, zusammen mit ausgeprägten vaskulären Läsionen (Teleangiektasien, fokale Blutungen).

Das Gehirn von Kindern ist empfindlicher als bei Erwachsenen. Funktionelle Defizite, wie eine Abnahme des Intelligenzquotienten (IQ), können zumindest

Onkologe 2018 $24: 797-801$ https://doi.org/10.1007/s00761-018-0400-5

(c) Der/die Autor(en) 2018

W. Dörr

\section{Spätfolgen nach Radiotherapie}

\section{Zusammenfassung}

Hintergrund. Eine kurative Strahlentherapie ist unumgänglich mit einem akzeptierten, geringen Risiko für (späte) Nebenwirkungen verbunden. Die klinische Manifestation von Strahleneffekten muss daher primär als Indikator für eine optimale Therapie angesehen werden.

Ziel der Arbeit. Ziel der vorliegenden Arbeit sind eine Darstellung der allgemeinen Pathogenese von Strahlenspätfolgen und die Charakterisierung der Latenzzeit. Späteffekte an ausgewählten, klinisch relevanten Risikoorganen werden beschrieben. Strahlenassoziierte Tumoren werden gesondert behandelt.

Material und Methoden. Diese Übersichtsarbeit beruht auf einer Zusammenstellung und Zusammenfassung der relevanten Literatur. Ergebnisse. Die Pathogenese von Strahlenspätfolgen ist komplex und beinhaltet Strahleneffekte an den Parenchymzellen der Organe, aber v. a. auch am Gefäßsystem und den Bindegewebsanteilen. Das Immunsystem trägt zur Reaktion bei. Folge ist letztendlich ein Funktionsausfall im exponierten Organvolumen. Die klinischen Konsequenzen einer Teilorganbestrahlung hängen vom Aufbau des betroffenen Organs ab. Die Latenzzeiten sind invers dosisabhängig und können Jahre bis zu vielen Jahrzenten betragen, was bei der Definition der Organtoleranz berücksichtigt werden muss. Sekundärtumoren nach Strahlentherapie haben häufig andere Ursachen. Die Inzidenz für eine Strahleninduktion liegt bei wenigen Prozent.

Schlussfolgerung. Die Möglichkeit einer signifikanten Verlängerung der Überlebenszeit bzw. Heilung des Primärtumors im Vergleich zu sehr langen Zeiträumen bis zur Manifestation eines strahleninduzierten Zweittumors rechtfertigt deshalb eindeutig die Strahlenanwendung.

Schlüsselwörter

Bestrahlungsfolgen - Spätkomplikation Pathogenese · Sekundärtumoren .

Strahleninduzierte Neoplasien

\section{Late effects after radiotherapy}

\section{Abstract}

Background. Curative radiotherapy is inevitably associated with an accepted low risk for (late) side effects. The clinical manifestation of radiation responses must thus primarily be considered as an indicator of optimal therapy.

Objective. The aim of the present work is presentation of the general pathogenesis of late radiation effects and the characterization of latency times. Late effects in selected clinically relevant organs at risk are described. Radiation-associated tumors are considered separately.

Materials and methods. This review is based on a compilation and summary of relevant literature.

Results. The pathogenesis of late radiation effects is complex and involves radiation effects on the parenchymal cells of the organs, but importantly also on the vascular system and connective tissue components. The immune system contributes to the reaction.
The result is ultimately a functional failure in the exposed organ volume. The clinical consequences of partial organ irradiation depend on the structure of the affected organ. The latency times are inversely dose dependent and can last for years to many decades, which must be taken into account when defining the organ tolerance. Secondary tumors after radiotherapy often have other causes. The incidence for radiation-induced tumors is in the range of a few percent. Conclusion. The possibility of significant prolongation of survival or healing of the primary tumor compared to very long periods until the manifestation of a radiation-induced secondary tumor therefore clearly justifies the application of ionizing radiation.

\section{Keywords}

Radiation effects - Long term adverse effects . Pathogenesis - Neoplasms, second primary . Neoplasms, radiation-induced 
teilweise der Strahlentherapie nach kombinierten Behandlungsprotokollen zugeschrieben werden. Veränderungen in der neurovaskulären Interaktion können zu Beeinträchtigungen des Lernens und des Gedächtnisses beitragen [26].

Die strahleninduzierten Veränderungen im Rückenmark ähneln denen im Gehirn in Bezug auf Latenzzeit, Histopathologie und Strahlentoleranz. Unter den (relativ) frühen Syndromen bei der Strahlentherapie von Kopf-Hals-Tumoren ist das Lhermitte-Zeichen eine häufig vorkommende, meist reversible demyelinisierende Reaktion, die sich einige Monate nach Beendigung der Behandlung entwickelt und Monate bis länger als ein Jahr anhält. Es kann bei Dosierungen ab 35 Gy in 2-Gy-Fraktionen auftreten, somit deutlich unter der Toleranz für die Strahlenmyelopathie, wenn lange Rückenmarkssegmente bestrahlt werden. Es besteht kein Zusammenhang mit der späteren Entwicklung einer Myelopathie.

Wie im Gehirn umfasst auch die späte Myelopathie (bis zur Querschnittslähmung) 2 Hauptsyndrome. Eine Demyelinisierung und Nekrose der weißen Substanztritt meist nach 6-18 Monaten auf. Der zweite Symptomenkomplex, mit einer Latenz von gewöhnlich 1 bis $>4$ Jahren, beruht hauptsächlich auf vaskulären Veränderungen.

\section{Herz und große Gefäße}

Die häufigste späte Strahlenfolge am Herzen ist eine Perikarditis mit unterschiedlich ausgeprägtem Perikarderguss. Diese beginnt relativ früh (6 Monate bis 2 Jahre). Sie ist meist asymptomatisch und heilt spontan bei der Mehrzahl der Patienten.

\section{》) Strahlenspätfolgen am Herzen treten nach sehr langen Latenzzeiten auf}

Die strahleninduzierte Kardiomyopathie tritt entweder als verminderter ventrikulärer Ausstoß oder als Leitungsblock auf; sie entsteht langsam über einen Zeitraum von 10-20 Jahren. Aktuelle Schätzungen von Dosen, die eine 50\%ige Komplika- tionswahrscheinlichkeit ergeben, liegen bei $50 \mathrm{~Gy}$ in 2 -Gy-Fraktionen $[5,21]$. Ein erhöhtes Risiko für eine ischämische Herzerkrankung wird nach mehr als 10 Jahren beobachtet.

Histopathologisch ist eine Spätschädigung des Myokards vorwiegend durch eine diffuse interstitielle und perivaskuläre Fibrose und Kardiomyozytenverlust charakterisiert. Vaskuläre Strahleneffekte tragen auch signifikant zum Myokardinfarkt nach Strahlenexposition des Herzens bei. Die (molekulare) Pathophysiologie dieser Effekte ist derzeit unklar.

In großen Blutgefäßen kann die Bestrahlung mit Dosen über 8 Gy Atherosklerose auslösen oder fördern.

\section{Auge}

In der Augenlinse wird bereits nach sehr niedrigen Strahlendosen - bei fraktionierter Bestrahlung im Bereich weniger Gy und bei einmaliger Exposition deutlich unter $1 \mathrm{~Gy}$ - eine Degeneration proliferierender Epithelzellen in der Äquatorzone beobachtet [5]. Schließlich entsteht eine subkapsuläre Strahlenkatarakt. Die Latenzzeiten liegen im Bereich von 6 Monaten bis zu mehreren Jahrzehnten. Anzumerken ist, dass Katarakte heute mit modernen Operationstechniken gut behandelt werden können.

Späte Effekte in den Tränendrüsen (Funktionsverlust) haben die Ausbildung eines "trockenen Auges“" zur Folge. Bereits nach moderaten Strahlendosen können diese zu chronischen Korneaulzerationen und letztendlich zum Verlust des Auges führen.

\section{Strahlenassoziierte Tumoren}

Für solide Sekundärtumoren nach Strahlentherapie kann die Latenz bis zu mehreren Jahrzehnten betragen [27]. Die aus der Strahlenempfindlichkeit der Allgemeinbevölkerung gegenüber homogener Ganzkörperexposition resultierenden Risikoschätzungen sind signifikant höher als die aus den epidemiologischen Studien resultierenden Werte [22, 24]. Der Grund hierfür liegt u. a. in Dosisinhomogenitäten im Körper und innerhalb einzelner Organe [17]. Derartige Inhomogenitäten verringern die Wahrscheinlichkeit einer Krebsinduktion signifikant.

Verschiedene Aspekte des Risikos für Zweitkrebserkrankungen nach Heilung eines ersten Tumors wurden von Trott und Dörr ausführlich diskutiert [24]. Hier können das Alter der Krebsüberlebenden, die fortgesetzte Exposition mit Karzinogenen und der genetische Hintergrund sowie andere Behandlungsmodalitäten (Chemotherapie) eine Rolle spielen.

Ein besserer Ansatz besteht darin, das Risiko von Zweitmalignomen durch Vergleich ihrer Häufigkeit in Patientenkohorten nach Behandlungen ohne (nur Operation) oder mit Strahlentherapie zu bestimmen. Voraussetzung ist aber, dass der Primärtumor eine hohe Frequenz und eine gute Prognose hat, die für beide Behandlungsmodalitäten ähnlich sind. Darüber hinaus sollte die Therapieentscheidung unabhängig von Faktoren sein, die Krebsrisiken betreffen. Diese Voraussetzungen werden weitgehend bei Gebärmutterhals- und Prostatamalignomen erfüllt. Zudem kann die Lokalisation des Sekundärtumors in Bezug auf diejenige des Primärmalignoms in symmetrischen Organen analysiert werden, insbesondere für primären Brust- und sekundären Lungenkrebs [22, 24].

\section{Mechanismen der Tumor- induktion}

Die klassische Form der Tumorinduktion durch Strahlentherapie ist die maligne Transformation einzelner Zellen. Bei Prostatatumoren [3] traten jedoch fast $50 \%$ der Zweitmalignome in den hohen Dosisvolumina (Harnblase, Rektum) auf. Ähnliches wurde für andere Tumorentitäten berichtet [26]. Wahrscheinlich liegt in diesen Regionen ein völlig anderer Mechanismus der Krebsinduktion vor $[8,24]$ : Hohe Strahlendosen führen häufig zu einer chronischen hyperproliferativen Gewebsreaktion mit progressiver Parenchymatrophie, mikrovaskulären Veränderungen, chronischer Fibrose und chronischen Entzündungen, die in ihrer Kombination präkanzeröse Läsionen darstellen.

Strahleninduzierte Sekundärtumoren manifestieren sich erst nach vielen Jah- 
ren bis Jahrzehnten. Das Risiko für radiogene Zweitkarzinome liegt nach radikaler Strahlentherapie bei den meisten Krebserkrankungen bei Erwachsenen bei sehr wenigen Prozent [24]. Für die Strahlentherapie maligner Erkrankungen im Kindesalter müssen diese Schätzungen erst definiert werden $[8,24]$. Im Gegensatz zum Sekundärtumorrisiko ist die Wahrscheinlichkeit einer (sehr) frühen Mortalität aufgrund fehlender Kontrolle des Primärtumors für die meisten Tumorentitäten wesentlich höher. In Anbetracht der negativen Auswirkungen einer suboptimalen Therapie rechtfertigt die Möglichkeit einer signifikanten Verlängerung der Überlebenszeit bzw. Heilung des Primärtumors eindeutig die Strahlenanwendung [8].

\section{Fazit für die Praxis}

\section{- Strahlenspätfolgen sind Indikatoren für eine optimale Therapie. \\ - Ein geringes Risiko muss akzeptiert werden. \\ - Die Pathogenese ist komplex und schließt neben dem Organparen- chym das Gefäßsystem und Bidege- websanteile ein. \\ - Das Immunsystem (Makrophagen) trägt zum Geschehen bei. \\ - Latenzzeiten sind invers dosisab- hängig, mit Konsequenzen für die Nachbeobachtungszeit. \\ - Bei Teilorganexposition definiert die Struktur des Organs die klinischen Konsequenzen. \\ - Das Risiko für strahleninduzierte Sekundärtumoren liegt im Bereich weniger Prozent.}

\section{Korrespondenzadresse}

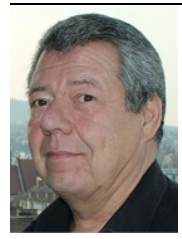

Univ.-Prof. Dr. med. vet. et rer. medic. habil. W. Dörr Klinik für Strahlentherapie, ATRAB - Applied and Translational Radiobiology, Medizinische Universität Wien Währinger Gürtel 18-20, 1090 Wien, Österreich wolfgang.doerr@ meduniwien.ac.at
Funding. Open access funding provided by Medical University of Vienna.

\section{Einhaltung ethischer Richtlinien}

Interessenkonflikt. W. Dörr gibt an, dass kein Interessenkonflikt besteht.

Dieser Beitrag beinhaltet keine von den Autoren durchgeführten Studien an Menschen oder Tieren.

Open Access Dieser Artikel wird unter der Creative Commons Namensnennung 4.0 International Lizenz (http://creativecommons.org/licenses/by/4.0/deed. de) veröffentlicht, welche die Nutzung, Vervielfältigung, Bearbeitung, Verbreitung und Wiedergabe in jeglichem Medium und Format erlaubt, sofern Sie den/die ursprünglichen Autor(en) und die Quelle ordnungsgemäß nennen, einen Linkzur Creative Commons Lizenz beifügen und angeben, ob Änderungen vorgenommen wurden.

\section{Literatur}

1. Bentzen SM (2006) Preventing or reducing late side effects of radiation therapy: radiobiology meets molecular pathology. Nat Rev Cancer 6:702-713

2. Bentzen SM, Constine LS, Deasy JO, Eisbruch A, Jackson A, Marks LB, Ten Haken RK, Yorke ED (2010) Quantitative Analyses of Normal Tissue Effects in the Clinic (QUANTEC): an introduction to the scientific issues. Int J Radiat Oncol Biol Phys 76(3Suppl):S3-9.https://doi.org/10.1016/j.jirobp. 2009.09.040

3. Brenner DJ, Curtis RE, Hall EJ, Ron E (2000) Second malignancies in prostate carcinoma patients after radiotherapy compared with surgery. Cancer 88:398-406

4. Clement $\mathrm{CH}$, StewartFA, International Commission on Radiological Protection (2012) ICRP statement on tissue reactions and early and late effects of radiation in normal tissues and organs: threshold doses for tissue reactions in a radiation protection context. Ann ICRP 11841(1):2012

5. Dörr W (2018) Pathogenesis of normal-tissue sideeffects. In: Joiner MC, Van der Kogel AJ (Hrsg) Basic Clinical Radiobiology, 5. Aufl. CRCPress, Abingdon, SKap 14 (im Druck)

6. Dörr W, Bertmann S, Herrmann T (2005) Radiation induced lung reactions in breast cancer therapy. Modulating factors and consequential effects. Strahlenther Onkol 181:567-573

7. DörrW,HendryJH(2001)Consequential lateeffects in normal tissues. Radiother Oncol 61:223-231

8. Dörr W, Herrmann T (2002) Cancer induction by radiotherapy: dose dependence and relation to irradiated volume. JRadiol Prot 22:A117-A121

9. Dörr W, Herrmann T (2003) Pathogenetic mechanisms of lung fibrosis. In: Nieder C, Milas L, Ang KK (Hrsg) Biological modification of radiation response. Springer, Berlin, S29-36

10. Dörr W, Herrmann T, Riesenbeck D (Hrsg) (2005) Prävention und Therapie von Nebenwirkungen in der Strahlentherapie. UNI-MED Science, Bremen

11. Dörr W, Van der Kogel AJ (2018) Normal tissue tolerance and the effect of dose inhomogeneities. In: Joiner MC, Van der Kogel AJ (Hrsg) Basic Clinical Radiobiology, 5. Aufl. CRC Press, Abingdon, S Kap 16 (im Druck)
12. Fajardo LF, Berthrong M, Anderson RE (2001) Radiation pathology. Oxford University Press, New York

13. Georg P, Boni A, Ghabuous A, Goldner G, Schmid MP, Georg D, Pötter R, Dörr W (2013) Time course of late rectal-and urinary bladder side effects after MRI-guided adaptive brachytherapy for cervical cancer. Strahlenther Onkol 189:535-540

14. Goldner G, Pötter R, Kranz A, Bluhm A, Dörr W (2011) Healing of late endoscopic changes in the rectum between 12 and 65 months after external beam radiotherapy. Strahlenther Onkol 187:202-205

15. Hakenjos L, Bamberg M, Rodemann HP(2000) TGFbeta1-mediated alterations of rat lung fibroblast differentiation resulting in the radiation-induced fibrotic phenotype. Int JRadiat Biol 76:503-509

16. Jaal J, Dörr W (2006) Radiation induced late damage to the barrier function of small blood vessels in mouse bladder. J Urol 176:2696-2700

17. Newhauser WD, Durante M (2011) Assessing the risk of second malignancies after modern radiotherapy. Nat Rev Cancer 11:438-448

18. Rodemann HP, Bamberg M (1995) Cellular basis of radiation-induced fibrosis. Radiother Oncol 35:83-90

19. Rubin P, Johnston CJ, Williams JP, McDonald S, Finkelstein JN (1995) A perpetual cascade of cytokines postirradiation leads to pulmonary fibrosis. Int J Radiat Oncol Biol Phys 33:99-109

20. Schaue D, Kachikwu EL, McBride WH (2012) Cytokines in radiobiological responses: a review. Radiat Res 178:505-523

21. Schultz-Hector S (1992) Radiation-induced heart disease: review of experimental data on dose response and pathogenesis. Int J Radiat Biol 61:149-160

22. Suit $H$, Goldberg S, Niemierko A, Ancukiewicz M, Hall E, Goitein M, Wong W, Paganetti H (2007) Secondary carcinogenesis in patients treated with radiation: a review of data on radiation-induced cancersin human, non-human primate, canineand rodent subjects. Radiat Res 167:12-42 (Erratum in Radiat Res 167:748)

23. Supiot S, Paris F (2012) Radiobiology dedicated to endothelium. Cancer Radiother 16:11-15

24. Trott KR, Dörr W (2018) Second cancers after radiotherapy. In: Joiner MC, Van der Kogel AJ (Hrsg) Basic Clinical Radiobiology, 5. Aufl. CRC Press, Abingdon, SKap 27 (im Druck)

25. Trott KR, Dörr W, Facoetti A, Hopewell J, Langendijk J, van Luijk P, Ottolenghi A, Smyth V (2012) Biological mechanisms of normal tissue damage: importance for the design of NTCP models. Radiother Oncol 105:79-85

26. Warrington JP, Ashpole N, Csiszar A, Lee YW, Ungvari Z, Sonntag WE (2013) Whole brain radiationinduced vascular cognitive impairment: mechanisms and implications. J Vasc Res 50:445-457

27. Welte B, Suhr P, Bottke D, Dörr W, Trott KR, Wiegel T (2010) Second malignancies in high-dose areas of previous tumor radiotherapy. Strahlenther Onkol 186:74-179

28. Yarnold J, Brotons MC (2010) Pathogenetic mechanisms in radiation fibrosis. Radiother Oncol 97:149-161 